\title{
High Pressure and Low Temperature Conducting Properties of Organic Semiconducting Metal-Anthraquinones
}

\author{
M. N. VIJAYASHREE, ${ }^{1,2}$ S. V. SUbramANYAM, ${ }^{2, *}$ and A. G. SAMUELSON ${ }^{3}$ \\ 'Jawaharlal Nehru Centre, Indian Institute of Science Campus, Bangalore, India, ${ }^{2}$ Department of Physics, \\ Indian Institute of Science, Bangalore 560012 , India, and ${ }^{3}$ Department of Inorganic \\ and Physical Chemistry, Indian Institute of Science, Bangalore 560 012, India
}

\begin{abstract}
SYNOPSIS
High pressure and low temperature conducting properties of 1,4-diamino anthraquinone, with metals such as cobalt, nickel, copper, and silver, are reported. The complexes formed are oxidatively and thermally stable and are insoluble in common organic solvents. They possess interesting conducting properties. All are semiconductors, showing conductivity in the range $1.98 \times 10^{-1}$ to $1.36 \times 10^{-6} \mathrm{~S} / \mathrm{cm}$, depending upon the metal atom present in the complex. Low temperature ( $300 \mathrm{~K}$ to $30 \mathrm{~K}$ ) and high pressure (up to $6 \mathrm{GPa}$ ) conductivity properties of these materials are reported. Copper complexes have been studied in detail, as they show interesting conducting properties. (C) 1993 John Wiley \& Sons, Inc.
\end{abstract}

\section{INTRODUCTION}

Conducting organic materials have received considerable attention. ${ }^{1,2}$ The exciting possibilities of creating metal-like conductivity, superconductivity, and unusual optical and magnetic properties have stimulated this interest. Several polymers, such as polyacetylene, ${ }^{3-6}$ Poly ( $p$-phenylene), ${ }^{7}$ poly (phenylenesulfide ), ${ }^{8}$ and polypyrrole ${ }^{9,10}$ have been found to be highly conductive when oxidized or reduced. Under ambient conditions, the highly reactive ions can react with moisture and/or oxygen, which reduces the conductivity. ${ }^{11,12}$ The introduction of less reactive species, such as transition metals, into the conduction path that was capable of exhibiting multiple oxidation states, was thought to be a method of producing an intrinsically stable conducting polymer. A transition metal could serve as an internal oxidant or reductant, so that the polymer would not require any external oxidizing or reducing agent to achieve a conducting state. Polymeric metal complexes, derived from 2,5 diamino 1,4-Benzene dithiol ${ }^{13}$ and tetrathiooxalates, ${ }^{14}$ fall into this category. We have synthesized several metal complexes

\footnotetext{
* To whom correspondence should be addressed.

Journal of Applied Polymer Science, Vol. 49, 2171-2177 (1993)

(C) 1993 John Wiley \& Sons, Inc. CCC 0021-8995/93/122171-07
}

of 1,4-diaminoanthraquinone, which exhibit interesting conductivity behavior. In order to understand the nature of these classes of compounds, we have investigated the low temperature conducting behavior of the conducting coordination oligomers.

The pressure effects have been widely used to provide insight into the basic character of the electronic conduction in molecular solids. As the pressure is applied on the organic conductors, both the mobility and carrier concentrations markedly increase. ${ }^{15}$ This occurs because of the increased tunnelling and hopping available to mobile electrons, since the pressure decreases the average distance between the molecules. ${ }^{16-18}$ The variation of resistivity with hydrostatic pressure has been studied up to $6 \mathrm{GPa}$.

\section{EXPERIMENTAL}

The starting material, 1,4-diaminoanthraquinone (Aldrich), is recrystallized several times from acetone before use. Other chemicals are of reagent grade.

$\mathrm{C}, \mathrm{H}, \mathrm{N}$-analysis is obtained from a Carolbra Strumentazine elemental analysis Mod 1106. Metal percentages are obtained from a Perkin-Elmer 2380 atomic absorption spectrophotometer. X-ray powder diffraction patterns are recorded on a Phillips PW 
$140 / 90$ scanning model, using Co $K_{\alpha}(\lambda=1.791 \AA)$ radiation and a $2 \mathrm{deg} / \mathrm{min}$ scanning rate. The powdered complex is made into pellets ( $8 \mathrm{~mm}$ dia, $2 \mathrm{~mm}$ thick ), under $4 \mathrm{kbar}$ pressure, and the electrical resistivity data are taken using a conventional four probe (Vanderpauw) DC technique, with increasing and decreasing temperatures, in the range $300-30 \mathrm{~K}$.

High pressure studies have been performed, using a $4 \mathrm{~mm}$ tip Bridgman anvil setup. Pressure is generated by a hydraulic press, supplied by AIMIL India Ltd., Bombay. For the precision control of the oil flow, a needle valve is provided. The oil pressure is measured by a Heise gauge $(0-1000 \mathrm{psi})$, with a resolution of $1 \mathrm{psi}$ ( pounds per square inch). The room temperature calibration of the generated pressure has been performed using the phase transitions of bismuth and ytterbium.

\section{Synthesis of Copper (II)/ 1,4- Diaminoanthraquinone Complex}

1,4-diaminoanthraquinone ( $1.00 \mathrm{gm})$ is added to a solution of copper (II) nitrate trihydrate $(0.769 \mathrm{gm})$, dissolved in dimethyl formamide $(25 \mathrm{~mL})$. The black precipitate formed is filtered and is washed successively with water, acetone, and methylene chloride several times, until the washings are colorless, to remove unreacted starting materials. The product is dried at $40^{\circ} \mathrm{C}$ in a vacuum oven for about $15 \mathrm{~h}$.

\section{Complex of Cobalt (II)/1,4- Diaminoanthraquinone}

Cobalt (II) chloride hexahydrate $(0.5 \mathrm{gm})$ and triethylamine $(0.84 \mathrm{gm})$ are added to a solution of 1,4 diaminoanthraquinone $(0.5 \mathrm{gm})$, dissolved in dimethylformamide ( $25 \mathrm{~mL}$ ). The black precipitate formed is washed with acetone, water, and meth- ylene chloride, until the washings are colorless, to remove the unreacted starting materials. The final product is dried at $40^{\circ} \mathrm{C}$ in a vacuum oven for $15 \mathrm{~h}$.

\section{Platinum (II)/1,4-Diaminoanthraquinone}

Recrystallized 1,4-diaminoanthraquinone ( $0.26 \mathrm{gm})$ is dissolved in dimethylformamide $(25 \mathrm{~mL})$. To this, platinum (II) chloride ( 0.36 ) is added and is left for $1 \mathrm{~h}$. The precipitate of the complex is washed with acetone, water, and methylene chloride, until the washings are colorless, and is dried under vacuum at $40^{\circ} \mathrm{C}$ overnight. The palladium complex is prepared in a similar manner, using palladium dichloride. Since the yield is poor, the data on these complexes could not be obtained.

Nickel, silver complexes, and complexes of copper, starting with copper (I) chloride and copper (II) chloride, are prepared using the method reported earlier. ${ }^{19}$

\section{RESULTS AND DISCUSSION}

The solubility of these complexes is extremely low, even in solvents such as DMSO, making the characterization difficult. The molecular weight could not be obtained for the same reason.

The elemental analysis and the corresponding empirical formulae of the complexes are obtained from $\mathrm{C}, \mathrm{H}, \mathrm{N}$, and metal percentages. The percentage of oxygen is calculated to be the remainder after the percentages of the other elements are determined. The details are shown in Table I.

The X-ray powder diffraction pattern of these complexes shows them to be polycrystalline with two major $d$-spacings common in all complexes (Figs. 1 and 2). The most intense diffraction peak corresponds to a $d$-spacing of $11 \AA$ and of $3.2 \AA$. The crystallite sizes, computed for these complexes using

Table I Elemental Assay and Empirical Formula for the 1,4-DAAQ Metal Complexes

\begin{tabular}{lllllll}
\hline $\begin{array}{l}\text { Metal } \\
\text { Salt }\end{array}$ & $\begin{array}{c}\mathrm{C} \\
\%\end{array}$ & $\begin{array}{c}\mathrm{H} \\
\%\end{array}$ & $\begin{array}{l}\mathrm{N} \\
\%\end{array}$ & $\mathrm{O}^{\mathrm{a}}$ & Metal \% & Emperical Formula \\
\hline $\mathrm{Cu}(\mathrm{I}) \mathrm{Cl}$ & 40.8 & 2.66 & 6.72 & 27.2 & 22.2 & $\mathrm{C}_{14} \mathrm{H}_{10} \mathrm{~N}_{2} \mathrm{O}_{4} \mathrm{Cu}_{1.4}$ \\
$\mathrm{CuCl}$ & & 3.15 & 8.0 & 27.25 & 12.50 & $\mathrm{C}_{14} \mathrm{H}_{10} \mathrm{~N}_{2} \mathrm{O}_{4.3} \mathrm{Cu}_{0.6}$ \\
$\mathrm{Cu}\left(\mathrm{NO}_{3}\right)_{2}$ & 48.0 & 2.77 & 9.6 & 21.13 & 18.50 & $\mathrm{C}_{14} \mathrm{H}_{10} \mathrm{~N}_{2.4} \mathrm{O}_{4.7} \mathrm{Cu}_{1.05}$ \\
$\mathrm{NiCl}_{2}$ & 49.1 & 2.60 & 7.74 & 23.72 & 17.80 & $\mathrm{C}_{14} \mathrm{H}_{10} \mathrm{~N}_{2.1} \mathrm{O}_{4.3} \mathrm{Ni}_{0.98}$ \\
$\mathrm{AgNO}_{3}$ & 47.2 & 2.52 & 7.99 & 24.49 & 23.30 & $\mathrm{C}_{14} \mathrm{H}_{10} \mathrm{~N}_{2.13} \mathrm{O}_{3.78} \mathrm{Ag}_{0.8}$ \\
$\mathrm{CoCl}_{2}$ & 41.7 & 3.47 & 8.22 & 26.08 & 12.18 & $\mathrm{C}_{14} \mathrm{H}_{10} \mathrm{~N}_{1.98} \mathrm{O}_{5.3} \mathrm{Co}_{0.73}$ \\
\hline
\end{tabular}

\footnotetext{
a Calculated to be the remainder after the percentages of the other elements are determined.
} 


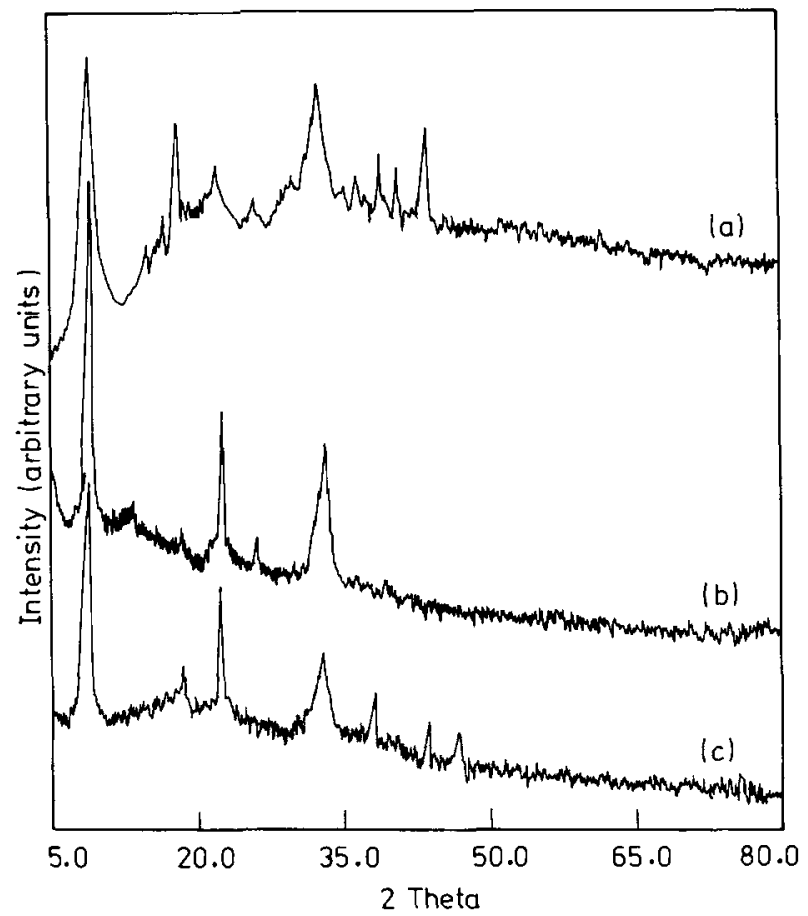

Figure $1 \mathrm{X}$-ray powder diffraction of Copper complex (a) $\mathrm{Cu}$ (II) nitrate, (b) $\mathrm{Cu}$ (I) chloride, and (c) $\mathrm{Cu}$ (II) chloride.

the Scherrer formula, ${ }^{20}$ are presented in Table II. This allows for computation of the average number of repeating units per crystallite, obtained using the crystallite size and $d$-spacing. ${ }^{21}$

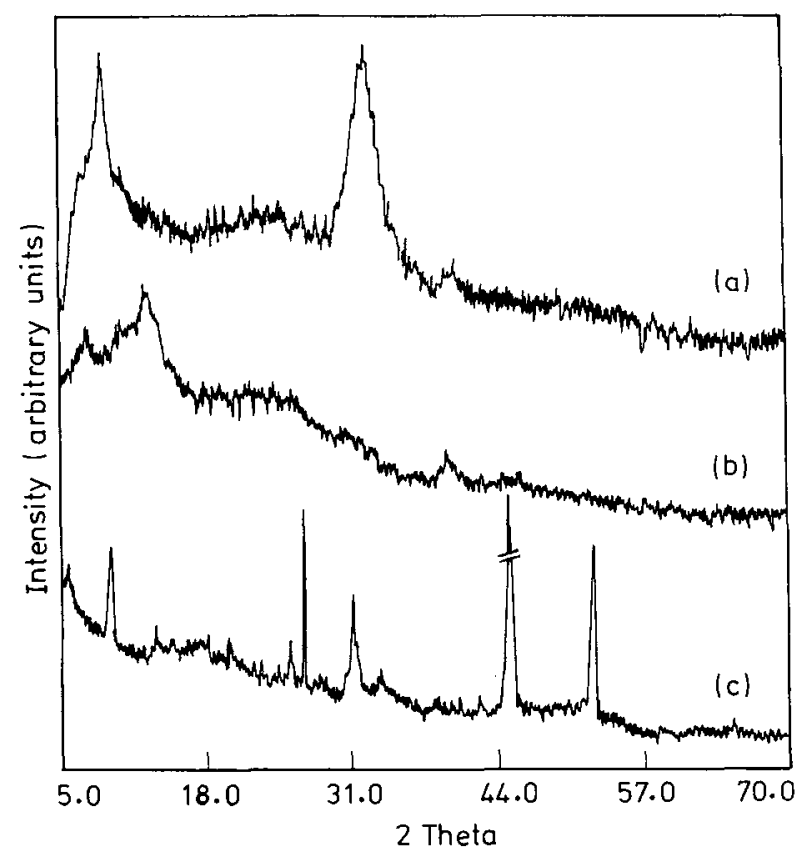

Figure 2 X-ray powder diffraction of (a) Ni-complex, (b) Co-complex, and (c) Ag-complex.
Table II Crystallite Size and Conductivity of 1,4-DAAQ Complexes

\begin{tabular}{lccc}
$\begin{array}{c}\text { Metal } \\
\text { Present }\end{array}$ & $\begin{array}{c}\text { Crystallite } \\
\text { size } \AA\end{array}$ & $\begin{array}{c}\text { Repeating } \\
\text { Units }\end{array}$ & $\begin{array}{c}\sigma \mathrm{S} / \mathrm{cm} \text { at } \\
300 \mathrm{~K}\end{array}$ \\
\hline $\mathrm{Cu}^{\mathrm{a}}$ & $60-40$ & $6-4$ & $1.98 \times 10^{-1}$ \\
$\mathrm{Cu}^{\mathrm{b}}$ & $74-120$ & $11-7$ & $9.15 \times 10^{-2}$ \\
$\mathrm{Cu}^{\mathrm{c}}$ & $94-90$ & $9-8$ & $1.3 \times 10^{-3}$ \\
$\mathrm{Ni}$ & $48-37$ & $6-4$ & $5.81 \times 10^{-2}$ \\
$\mathrm{Ag}$ & $162-131$ & $16-13$ & $1.85 \times 10^{-3}$ \\
$\mathrm{Co}$ & $42-21$ & $5-4$ & $1.15 \times 10^{-4}$ \\
$\mathrm{Pt}$ & & & $1.53 \times 10^{-5}$ \\
$\mathrm{Pd}$ & & & $1.31 \times 10^{-6}$ \\
\hline
\end{tabular}

${ }^{a}$ Copper complex obtained from copper (I) chloride.

${ }^{b}$ Copper complex obtained from copper (II) nitrate.

${ }^{\mathrm{c}}$ Copper complex obtained from copper (II) chloride.

A tentative structure of the polymeric the metal complex was proposed by Rickel. ${ }^{19}$ This structure involves sheets of 1,4-DAAQ, held together as shown in Scheme I below

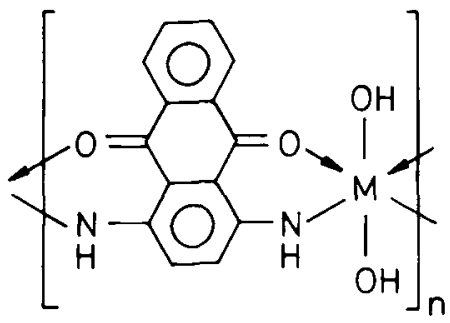

Proposed structure of the complex where $n=4-16$

Polycrystalline samples of cobalt, nickel, copper, silver, palladium, and platinum are prepared and the variation of conductivity with different metal atoms is given in Table II. The conductivity of the copper complex, being the highest, seems to indicate the tendency for copper to form square planar geometry as compared to cobalt and nickel. Furthermore, there appears to be good correlation between the number of $d$-electrons and the conductivity. Complexes of palladium and platinum show high resistance as compared to those of nickel. This may be due to the $5 d^{8}$ and $6 d^{8}$ configuration and the tendency of these metal atoms to form octahedral complexes. Formation of such octahedral complexes would lead to three dimensional networks.

Copper complexes, obtained from different starting copper salts, that is, copper (I) chloride, copper (II) nitrate, and copper (II) chloride, exhibit different thermal conductivities. This could be explained on the basis of the amount of copper that 


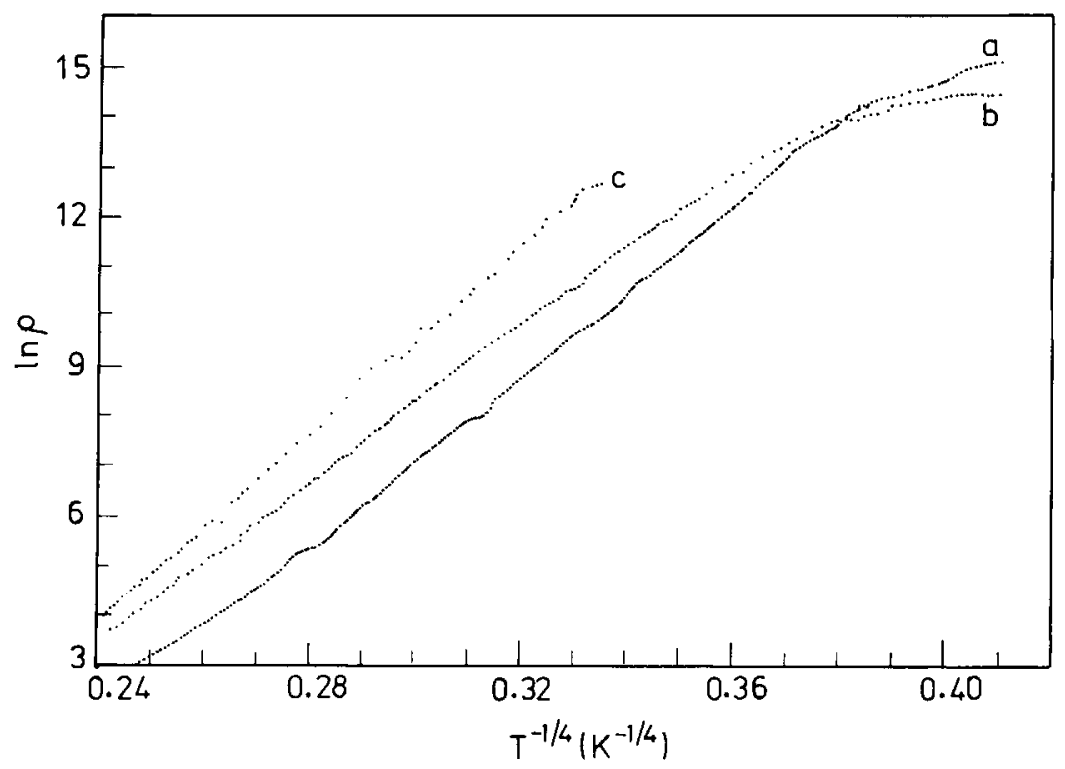

Figure 3 Ln $\rho$ vs. $(T)^{-1 / 4}$ plots of copper complexes prepared from (a) copper (I) chloride, (b) copper (II) nitrate, and (c) copper (II) chloride.

has gone into the system in obtaining the complex. It is clear from Table II and Figure 3 that the complexes containing more copper have greater conductivity. Surprisingly, complexes prepared with copper chloride show a complete absence of chlorine, as confirmed by several tests for chlorine.

For silver and cobalt complexes, measurements are performed down to $77 \mathrm{~K}$. In these complexes, the resistance is of the order of Mega ohms or larger below $77 \mathrm{~K}$. For other complexes, the measurements are carried out until $30 \mathrm{~K}$. In order to interpret the data, $\ln (\rho)$ has been plotted against the reciprocal of absolute temperature (Fig. 4). For semiconducting materials, these plots should yield straight lines over the given temperature range, obeying the equation $\rho=\rho_{0} \exp (E / 2 k T)$, where $\rho$ is the resistivity at absolute temperature $T, \rho_{0}$ is a constant, and $E$ is the energy gap. In this case, it was found that such a simple relationship does not hold good.

Several models have been proposed to account for the electronic properties of the various groups of polycrystalline materials. ${ }^{22}$ However, the concept of localized states at the Fermi level is common to all models; the electronic charge transport critically

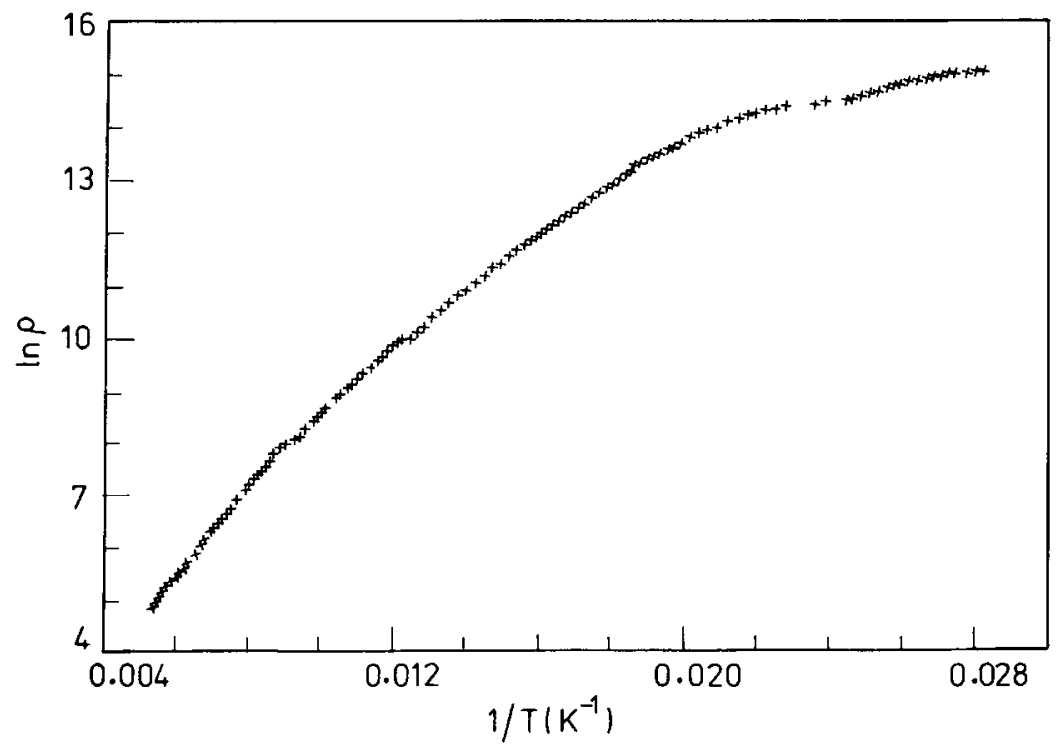

Figure 4 Low temperature resistivity behavior of copper complex. 


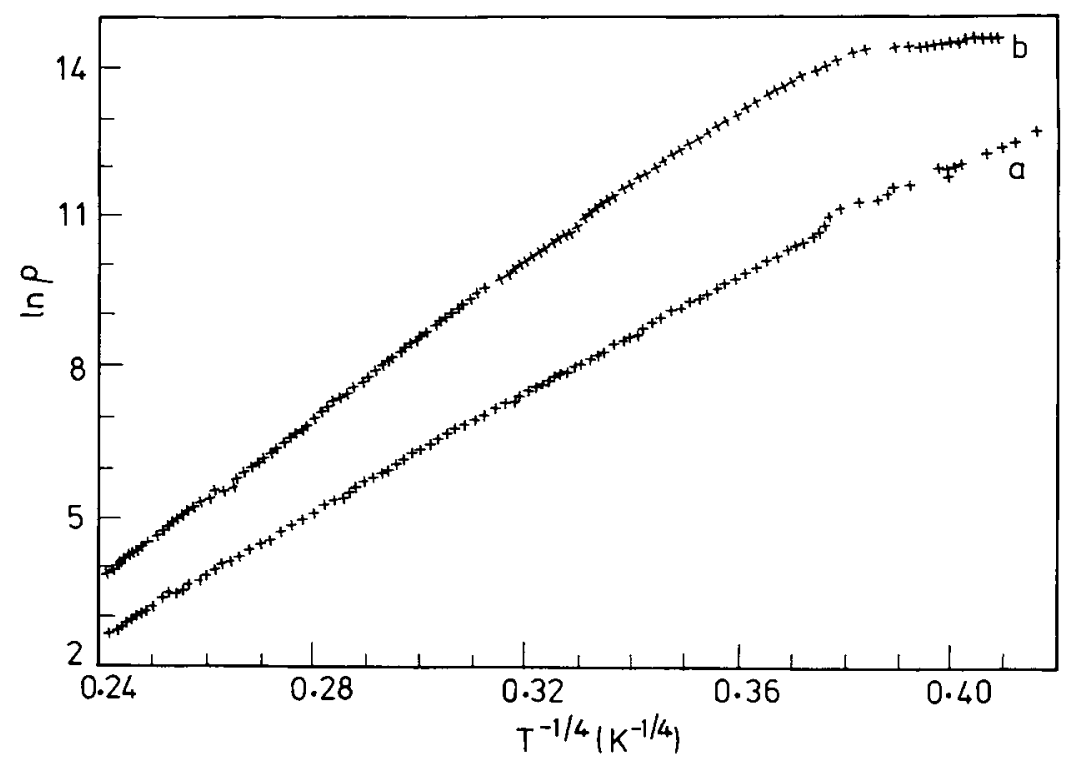

Figure $5 \operatorname{Ln} \rho$ vs. $(T)^{-1 / 4}$ of nickel and copper complexes.

depends on it. The semiconducting behavior is driven by the minimal activation energy for free charge carrier to go through the mobility gap. A large enough thermal agitation is necessary for this process to hold, and at low temperatures, other mechanisms become important. The $d c$ conductivity does not follow a simple exponential law. As the temperature decreases, it is postulated that conduction proceeds through two kinds of hopping among localized states near the Fermi level: (1) thermallyassisted tunneling between the localized gap states near the mobility edges and (2) tunneling conduction near the Fermi level (when tunneling is restricted to seek centers, which lie energetically closer and within the range $k T$ ). This is the VariableRange-Hopping process, described by Mott and Davis, ${ }^{23}$ and this process has the following relation for de conductivity

$$
\sigma=\text { Const. }\left[\left(T_{0} / T\right)^{1 / 1+n}\right]
$$

where $n=3,2$, or 1 , depending on the dimensionality of the conducting system. $T_{0}$ is a constant, which

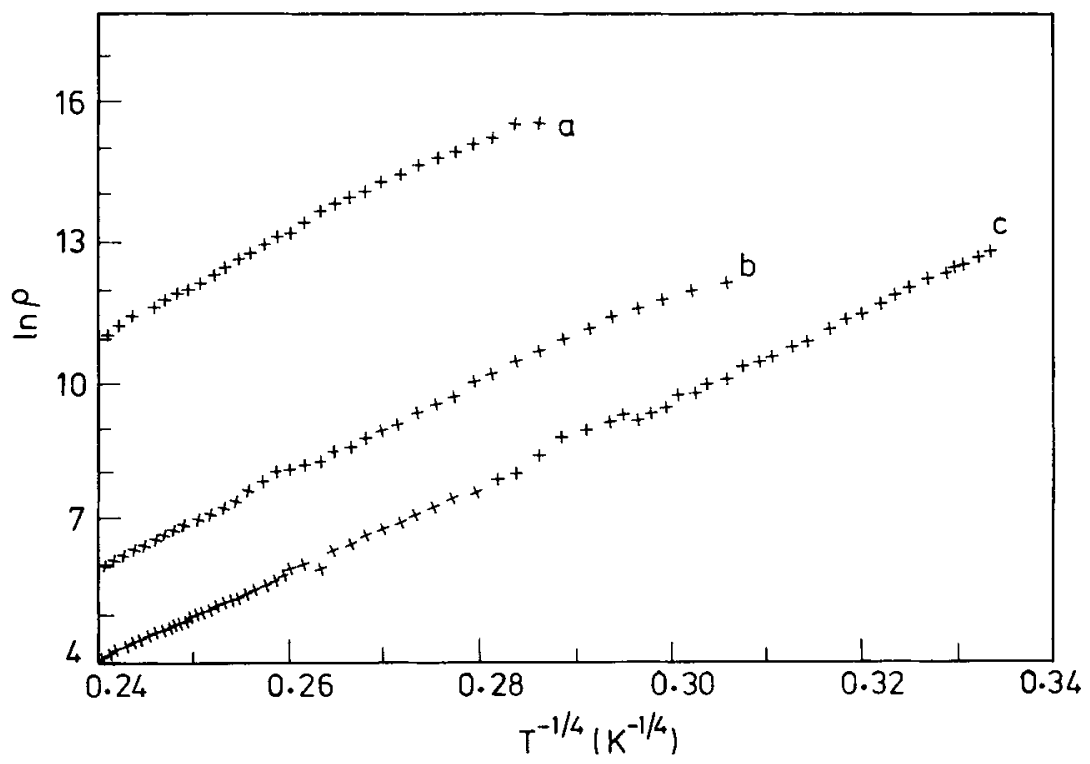

Figure 6 Ln $\rho$ vs. $(T)^{-1 / 4}$ plots. (a) cobalt complex, (b) silver complex, and (c) copper (II) chloride complex. 


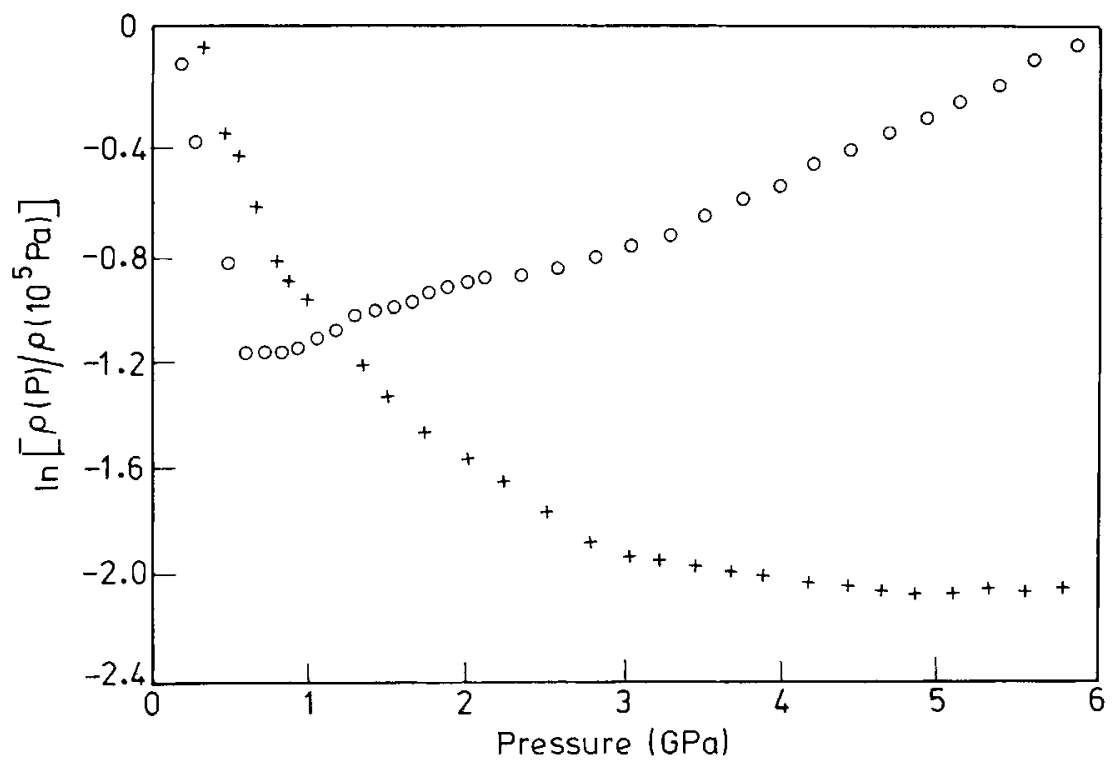

Figure 7 Normalized resistivity as a function of pressure. $(\odot)$ copper complex and $(+)$ cobalt complex.

depends on the details of the model, is always inversely proportional to the density of states at the Fermi level, and is assumed to be energy independent.

We find that the data is in general agreement with the above model. The conductivity measurements of these complexes show that the temperature dependence satisfies the relation $\ln (\rho) \propto T^{-1 / 4}$ (Figs. 5 and 6). Thus, the carrier transport process, studied by the temperature dependence of conductivity, shows that conduction is not governed by carriers excited fully to the extended states, but by the hopping of carriers excited to the localized states near the mobility edge.

Pressures up to $6 \mathrm{GPa}$ have been applied to study the variation of resistivity of the sample with pressure. The resistivity of the complexes generally decreases with pressure in the beginning and later remains constant. This behavior of resistivity variation with pressure has been observed in many organic conductors, ${ }^{24}$ and has been explained on the basis of the molecular orbital overlap picture, where

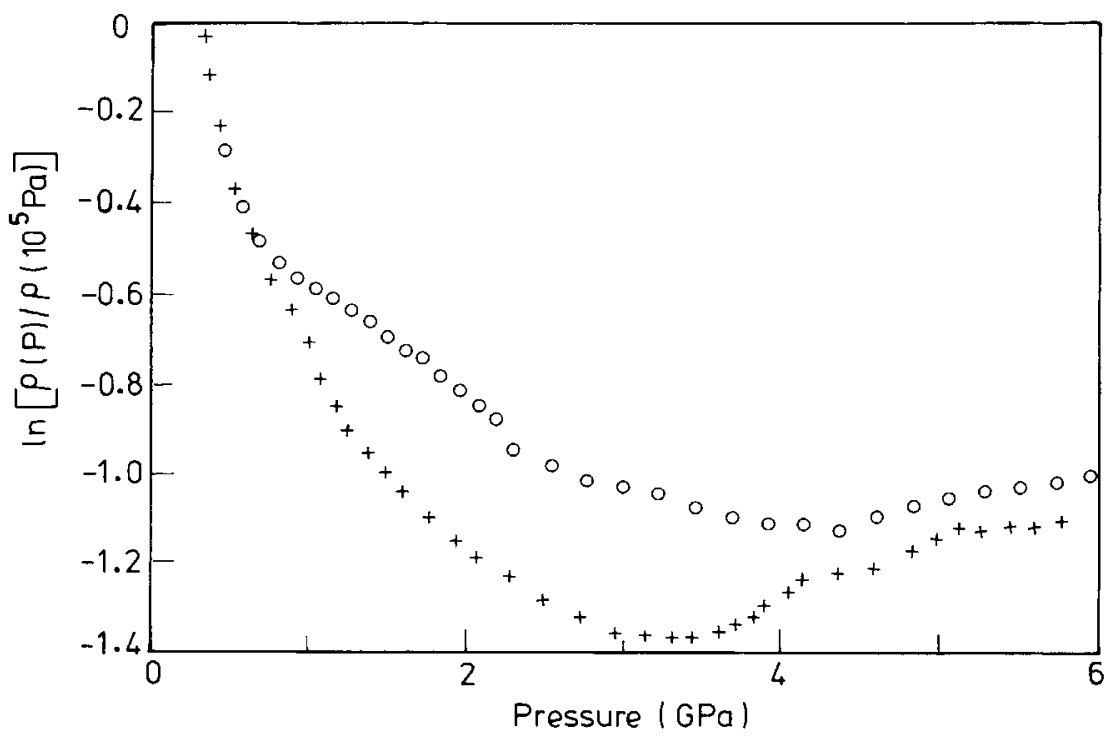

Figure 8 Normalized resistivity as a function of pressure. $(\odot)$ silver complex and (+) nickel complex. 
with increasing pressure, the overlap between adjacent molecules increases, resulting in enhanced conductivity. As the pressure is further increased, the coulomb repulsive forces result in the levelling off of the overlapping. If the steric hinderances and structural distortion become important, the resistivity may slightly increase at high pressures. This is the general behavior observed in the cobalt complex (Fig. 7), the silver complex (Fig. 8), and the nickel complex (Fig. 8). For the copper complex also, somewhat similar behavior is observed, except that the steric hinderances start dominating at much lower pressures. Similar behavior of resistivity with pressure at high pressures is observed by Bentley and Drikamer ${ }^{25}$ in many alkaline metals and organic semiconductors.

In conclusion, the complexes of 1,4-diaminonathraquinone, formed with cobalt, nickel, copper, and silver, are semiconducting, and exhibit variable range hopping transport at low temperatures $(30 \mathrm{~K}$ to $300 \mathrm{~K}$ ) and upon the application of hydrostatic pressure, conductivity increases and reaches a saturation value.

The authors are grateful to the Department of Science and Technology, Government of India, New Delhi, India, for financial assistance.

\section{REFERENCES}

1. Proceedings of the O.J.I. International Seminar on Organic Semiconductors-40 Years. Molecular Crystals and Liquid Crystals Incorporating Nonlinear Optics. 171, 1-356 (1989).

2. International Conference on Science and Technology of Synthetic Metals, (ICSM '90) Sept. 2-7 (1990).

3. H. Shirakawa, T. Ito, and S. Ikeda, Polym. J., 4, 460 (1973).

4. H. Shirakawa, E. J. Louis, A. G. Macdiarmid, C. K. Chinag, and A. J. Heeger, J. Chem. Soc. Chem. Commun., 578 (1977).

5. L. W. Shacklette, R. R. Chance, D. M. Ivory, G. G. Muller, and R. H. Baughman, Synth. Met., 1, 307 (1980).
6. L. W. Shacklette, R. L. Elsenbaumer, R. R. Chance, H. Eckharot, J. E. Frommer and R. H. Baughman, J. Chem. Phys., 75, 1919 (1981).

7. K. K. Kanazawa, A. T. Daiz, W. D. Gill, P. M. Grant, G. B. Street, G. P. Gardini and J. F. Kwak, Synth. Met., 1, 329 (1980).

8. D. Wohrle, Adv. Polym. Sci., 47, 50 (1983).

9. R. S. Norh, P. M. Kuznesof, K. J. Wynne, M. E. Kenney, and P. G. Srebenmann, J. Am. Chem. Soc., 103, 4371 (1981).

10. K. F. Schoch, B. R. Kundalkar, and T. J. Marks, J. Am. Chem. Soc., 101, 7071 (1979).

11. Oh-Kilmin, T. H. Yoon, and D. Mcdermitt, J. Chem. Soc. Chem. Commun., 11, 740 (1989).

12. Wegner and G. Angew, Chem. Int. Ed. Engl., 20, 361 (1981).

13. J. E. Frommer, Acc. Chem. Res., 2, 19 (1986).

14. G. E. Holdcroft and A. E. Underhill, Synth. Met., 10, 427 (1985).

15. S. Minomura, Ed., Solid State Physics under Pressure, D. Reidel, 'Tokyo, 1985.

16. H. A. Pohl, A. Rembaum, and A. Henry, J. Am. Chem. Soc., 84, 2699 (1962).

17. Y. Harada, Y. Maruyama, and I. Shirotani, Bull. Chem. Soc. Jpn., 37, 1378 (1964).

18. R. S. Bradly, J. C. Grace, and D. C. Monro, Trans Farady Soc., 46, 776 (1962).

19. G. K. Rickel, Macromolecules, 22, 1517 (1989).

20. B. D. Cullity, in E. Reading, Elements of X-Ray Diffraction, 2nd Ed, Addison-Wesley, Massachusetts (1978).

21. K. Tanaka, M. Ueda, T. Koike, T. Yamabe, and S. Yata, Synth. Metal, 25, 265 (1988).

22. M. H. Cohen, H. Fritzsche, and S. R. Ovslinsky, Phys. Rev. Lett., 22, 1065 (1969).

23. N. F. Mott and E. A. Davis, Electronic Processes in Noncrystalline Materials, 2nd Ed., Oxford University, Oxford, 1979.

24. K. Arai, K. Kumata, K. Kadota, K. Yamato, H. Namikawa, and S. Saito, J. Non-Crysta. Solids, 13, 131 (1973).

25. W. H. Bentley and H. G. Drikamer, J. Chem. Phy., 42, 1573 (1965).

Received September 15, 1992

Accepted January 25, 1993 